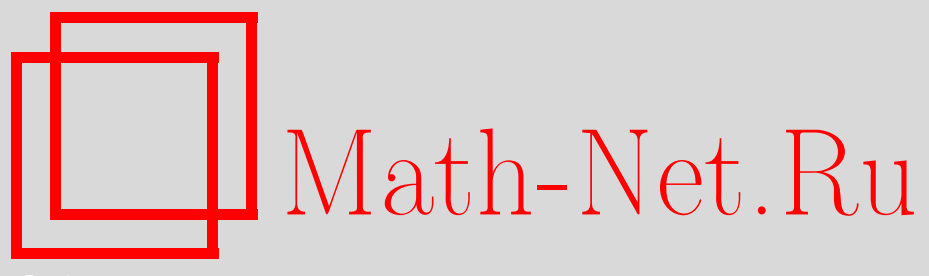

Д. Ф. Дигор, П. Ентел, В. А. Москаленко, Н. М. Плакида, Особенности парного взаимодействия в четырехзонной модели Хаббарда, ТМФ, 2006, том 149, номер 1, 99110

DOI: https://doi.org/10.4213/tmf3834

Использование Общероссийского математического портала Math-Net.Ru подразумевает, что вы прочитали и согласны с пользовательским соглашением http://www.mathnet.ru/rus/agreement

Параметры загрузки:

IP: 18.209 .158 .208

26 апреля 2023 г., 16:58:22

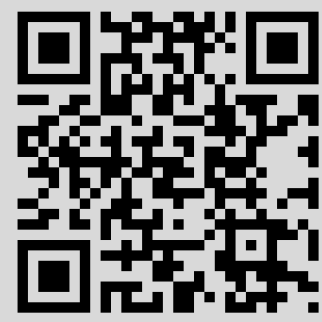




\title{
ОСОБЕННОСТИ ПАРНОГО ВЗАИМОДЕЙСТВИЯ В ЧЕТЫРЕХЗОННОЙ МОДЕЛИ ХАББАРДА
}

\begin{abstract}
Рассмотрена четырехзонная модель высокотемпературных сверхпроводников, содержащая $3 d_{x^{2}-y^{2}}$ и $4 s$-электроны меди и $2 p_{x^{-}}$и $2 p_{y}$-электроны кислорода. Предполагается сильное кулоновское отталкивание $3 d$-электронов меди. Выполнена диагонализация кислородной подсистемы и переход к представлению Ванье для кластера $\mathrm{CuO}_{2}$. Изучены свойства локальной ячеечной модели и определены такие ее низшие по энергии состояния, как одночастичный дублет и двухчастичные синглетные и триплетные состояния. Рассмотрены процессы делокализации фермионов ячейки, вызванные их туннелированием между узлами меди. Получена система уравнений типа Дайсона для перенормированных одночастичнных функций Грина и обсуждена возможность ее упрощения.
\end{abstract}

Ключевые слова: сильно коррелированные электронные системы, высокотемпературные сверхпроводники.

\section{1. ВВЕДЕНИЕ}

С момента открытия высокотемпературной сверхпроводимости (ВТСП) прилагались большие усилия для разработки простой теоретической модели, описывающей основные особенности этого явления.

В настоящее время общепринятым является положение об ответственности $\mathrm{CuO}_{2}$ плоскости купратов за сверхпроводящий фазовый переход. Мы исходим из модели сильной связи для электронных орбит этой плоскости и в качестве основного параметра теории рассматриваем сильное кулоновское отталкивание $d$-электронов меди, а также перескоки носителей заряда между ближайшими соседними узлами меди и кислорода.

* Институт прикладной физики АН Республики Молдова, Кишинев, Молдова.

E-mail: statphys@asm.md

${ }^{\dagger}$ Universität Duisburg-Essen, Institut für Fyzik, Duisburg, 47048, Lotharstrasse 1, Germany. E-mail: entel@thp.Uni-Duisburg.de

‡Объединенный институт ядерных исследований, Дубна, Россия.

E-mail: moskalen@theor.jinr.ru; plakida@theor.jinr.ru 


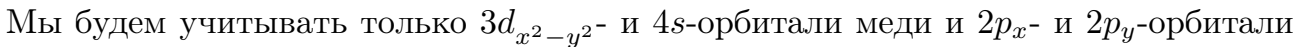
кислорода. Вакуумному состоянию соответствуют заполненные $3 d-, 4 s$ - и $2 p$-оболочки.

Отметим, что имеется значительное число статей и обзоров последних лет, посвященных теории ВТСП [1], в которых можно найти дальнейшие ссылки. Не вдаваясь в детали различных теоретических моделей, отметим, что все они указывают на существенную роль спиновых, зарядовых и парных флуктуаций в установлении явления сверхпроводимости и подчеркивают роль двухэлектронного обмена между различными орбиталями, принадлежащими разным атомам [2] или одному и тому же атому [3].

Цель настоящей работы состоит в том, чтобы изучить особенности четырехзонной модели купратов, принимая во внимание указанные выше орбитали меди и кислорода.

В разделе 2 формулируется $d-p-s$-модель, свойства которой изучаются в следующих разделах. Раздел 3 посвящен исследованию электронного спектра атомного предела ячеечного гамильтониана, тогда как в разделе 4 рассматривается процесс делокализации носителей заряда, происходящий благодаря межъячеечным туннельным процессам, и с этой целью развита соответствующая термодинамическая теория возмущений. В разделе 5 приводятся основные выводы.

\section{2. $d-p-s$-МОДЕЛЬ ХАББАРДА}

Гамильтониан данной модели имеет вид [2], [3]

$$
H=H_{d}+H_{p}+H_{s}+H_{d-p}+H_{s-p},
$$

где

$$
\begin{aligned}
H_{d} & =\epsilon_{d} \sum_{i \sigma} n_{i \sigma}^{d}+U \sum_{i} n_{i \uparrow}^{d} n_{i \downarrow}^{d}, \\
H_{p} & =\epsilon_{p} \sum_{l \sigma} n_{l \sigma}^{p}+t_{p p} \sum_{\left\langle l, l^{\prime}\right\rangle, \sigma} p_{l \sigma}^{+} p_{l^{\prime} \sigma} S_{l l^{\prime}}, \\
H_{s} & =\epsilon_{s} \sum_{i \sigma} n_{i \sigma}^{s}, \\
H_{d-p} & =t_{d p} \sum_{\langle i, l\rangle, \sigma}\left(d_{i \sigma}^{+} p_{l \sigma}+\text { э.c. }\right) S_{i l}, \\
H_{s-p} & =t_{s p} \sum_{\langle i, l\rangle, \sigma}\left(s_{i \sigma}^{+} p_{l \sigma}+\text { э.c. }\right) S_{i l}^{\prime} .
\end{aligned}
$$

Здесь $d_{i \sigma}\left(d_{i \sigma}^{+}\right), p_{l \sigma}\left(p_{l \sigma}^{+}\right)$и $s_{i \sigma}\left(s_{i \sigma}^{+}\right)$- операторы уничтожения (порождения) соответственно $d$-, $p$ - и $s$-дырок, расположенных на узлах меди $\vec{i}$ и кислорода $\vec{l}$, соответственно, и обладающих спином $\sigma$. Узлы меди образуют квадратную решетку в плоскости $\mathrm{CuO}_{2}$ с постоянной решетки $a$, тогда как координаты узлов кислорода в этой плоскости равны $\vec{l}=\vec{i} \pm(a / 2) \hat{x}, \vec{l}=\vec{i} \pm(a / 2) \hat{y}$, где $\hat{x}$ и $\hat{y}$ - единичные векторы двух координатнных осей элементарной ячейки $\mathrm{CuO}_{2}$. Плотности числа дырок равны $n_{i \sigma}^{d}=d_{i \sigma}^{+} d_{i \sigma}, n_{i}^{d}=n_{i \uparrow}^{d}+n_{i \downarrow}^{d}, n_{l \sigma}^{p}=p_{l \sigma}^{+} p_{l \sigma}, n_{l}^{p}=n_{l \uparrow}^{p}+n_{l \downarrow}^{p}$. Аналогичное 
определение имеется для $s$-дырок. Здесь $\epsilon_{d}, \epsilon_{p}$ и $\epsilon_{s}$ - одноузельные энергии дырок на соответствующих ионах, отсчитанные от химического потенциала системы: $\epsilon_{\alpha}=\bar{\epsilon}_{\alpha}-\mu, \alpha=d, p, s ; U-$ энергия кулоновского отталкивания дырок на узлах меди. Интегралы гибридизации состояний $t_{d p}, t_{p p}$ и $t_{s p}$ умножаются на специально введенные фазовые факторы $S_{i l}, S_{l l^{\prime}}$ и $S_{i l}^{\prime}$, равные \pm 1 . Выбор этих факторов для двух соседних орбиталей соответствует выбору, предложенному в работах [2], [3].

Имея в виду этот выбор, мы представим $d-p$ - и $s-p$-гибридизационные слагаемые (2) в виде

$$
\begin{aligned}
& 2 t_{d p} \sum_{i \sigma}\left(d_{i \sigma}^{+} \beta_{i \sigma}+\beta_{i \sigma}^{+} d_{i \sigma}\right), \\
& 2 t_{s p} \sum_{i \sigma}\left(s_{i \sigma}^{+} \alpha_{i \sigma}+\alpha_{i \sigma}^{+} s_{i \sigma}\right),
\end{aligned}
$$

где операторы уничтожения кластеров $\alpha_{i \sigma}$ и $\beta_{i \sigma}$ имеют вид

$$
\begin{aligned}
& \alpha_{i \sigma}=\frac{1}{2}\left[p_{\vec{i}+\frac{a}{2} \hat{x}, \sigma}^{x}-p_{\vec{i}+\frac{a}{2} \hat{y}, \sigma}^{y}+p_{\vec{i}-\frac{a}{2} \hat{x}, \sigma}^{x}-p_{\vec{i}-\frac{a}{2} \hat{y}, \sigma}^{y}\right] \\
& \beta_{i \sigma}=\frac{1}{2}\left[p_{\vec{i}+\frac{a}{2} \hat{x}, \sigma}^{x}-p_{\vec{i}+\frac{a}{2} \hat{y}, \sigma}^{y}-p_{\vec{i}-\frac{a}{2} \hat{x}, \sigma}^{x}+p_{\vec{i}-\frac{a}{2} \hat{y}, \sigma}^{y}\right] .
\end{aligned}
$$

Эти коллективные моды ячейки $\mathrm{CuO}_{2}$ обладают различными групповыми свойствами симметрии. Орбиталь $\beta_{i \sigma}$ была введена в работе Жанга и Райса [4] (см. также [5]), где было отмечено ее значение для образования локальных синглетных и триплетных состояний между дыркой на меди и допированными дырками на кислороде. Было показано, что существует локальный синглет между дыркой на центральном ионе $\mathrm{Cu}^{2+}$ и дыркой на четырех окружающих атомах кислорода, который имеет значительную энергию связи и движется по решетке, подобно дыркам в эффективной однозонной модели Хаббарда. Жанг и Райс высказали предположение о возможности описания низкоэнергетических свойств $d-p$-модели на основе ее редукции к более простой эффективной однозонной модели. В работах [6] приведена довольно подробная библиография статей в этой области.

Как будет видно из дальнейшего, эта концепция играет важную роль и в нашем случае четырехзонной модели. Далее мы следуем работам [6], в которых была показана целесообразность первоначальной диагонализации кислородного гамильтониана и с этой целью были введены диагонализующие $a$ - и $b$-фермионы по формулам

$$
\begin{aligned}
& p_{\vec{k} \sigma}^{x}=\frac{i}{\sqrt{2}}\left[\operatorname{Sgn}\left(S_{x}(\vec{k})\right) a_{\vec{k} \sigma}-\operatorname{Sgn}\left(S_{y}(\vec{k})\right) b_{\vec{k} \sigma}\right], \\
& p_{\vec{k} \sigma}^{y}=-\frac{i}{\sqrt{2}}\left[\operatorname{Sgn}\left(S_{y}(\vec{k})\right) a_{\vec{k} \sigma}+\operatorname{Sgn}\left(S_{x}(\vec{k})\right) b_{\vec{k} \sigma}\right],
\end{aligned}
$$

где $S_{x}(\vec{k})=\sin \left(k_{x} a / 2\right), S_{y}(\vec{k})=\sin \left(k_{y} a / 2\right)$.

В результате диагонализации получаем

$$
\begin{gathered}
H_{p}^{0}=\sum_{\vec{k}, \sigma}\left\{\epsilon_{a}(\vec{k}) a_{\vec{k} \sigma}^{+} a_{\vec{k} \sigma}+\epsilon_{b}(\vec{k}) b_{\vec{k} \sigma}^{+} b_{\vec{k} \sigma}\right), \\
\epsilon_{a, b}(\vec{k})=\epsilon_{p} \pm 4 t_{p p}\left|S_{x}(\vec{k}) S_{y}(\vec{k})\right| .
\end{gathered}
$$


Таким образом, диагонализация кислородных дырок приводит к появлению двух кислородных подзон, одна из которых $\left(\epsilon_{a}(\vec{k})\right)$ ниже, а другая $\left(\epsilon_{b}(\vec{k})\right)$ выше кислородного локального уровня.

Переход к ортогонализованным на узлах меди ячеечным амплитудам Ванье имеет вид

$$
a_{i \sigma}=\frac{1}{\sqrt{N}} \sum_{\vec{k}} a_{\vec{k} \sigma} e^{-i \vec{k} \vec{R}_{i}}
$$

Аналогичное уравнение имеет место для амплитуды $b_{i}$.

Ячеечные амплитуды (3) в представлении Ванье можно записать как

$$
\begin{aligned}
& \beta_{i \sigma}=\sum_{j}\left[\lambda_{a}(i-j) a_{i \sigma}+\lambda_{b}(i-j) b_{i \sigma}\right], \\
& \alpha_{i \sigma}=\sum_{j}\left[\bar{\lambda}_{a}(i-j) a_{i \sigma}+\bar{\lambda}_{b}(i-j) b_{i \sigma}\right] .
\end{aligned}
$$

Как следует из работ [6], новые межъячеечные амплитуды туннелирования $\lambda$ и $\bar{\lambda}$ оказываются отличными от нуля не только для ближайших соседних узлов меди, хотя и уменьшаются довольно быстро с расстоянием.

В новом ячеечном представлении четырехзонный гамильтониан Хаббарда приобретает вид

$$
H=H^{0}+H^{\prime}, \quad H^{0}=\sum_{i} H_{i}^{0},
$$

где одноячеечный локальный вклад равен

$$
H^{0}=\epsilon_{d} \sum_{\sigma} n_{\sigma}^{d}+U n_{\uparrow}^{d} n_{\downarrow}^{d}+\epsilon_{a} \sum_{\sigma} n_{\sigma}^{a}+\epsilon_{b} \sum_{\sigma} n_{\sigma}^{b}+\epsilon_{s} \sum_{\sigma} n_{\sigma}^{s}+t_{a, d} \sum_{\sigma}\left(d_{\sigma}^{+} a_{\sigma}+a_{\sigma}^{+} d_{\sigma}\right) .
$$

Здесь для простоты опущен индекс $i$. Возмущение $H^{\prime}$ имеет вид

$$
\begin{aligned}
H^{\prime}= & \sum_{j \neq i, \sigma}\left[d_{i \sigma}^{+}\left(t_{d, a}(i-j) a_{j \sigma}+t_{d, b}(i-j) b_{j \sigma}\right)+\text { э.c. }\right]+ \\
& +\sum_{j \neq i, \sigma}\left[s_{i \sigma}^{+}\left(t_{s, a}(i-j) a_{j \sigma}+t_{s, b}(i-j) b_{j \sigma}\right)+\text { э.c. }\right]+ \\
& +\sum_{j \neq i, \sigma}\left[a_{i \sigma}^{+} t_{a, a}(i-j) a_{j \sigma}+b_{i \sigma}^{+} t_{b, b}(i-j) b_{j \sigma}\right]
\end{aligned}
$$

где использованы обозначения

$$
\begin{aligned}
t_{d, a}(i-j) & =2 t_{d, p} \lambda_{a}(i-j), \\
t_{d, b}(i-j) & =2 t_{d, p} \lambda_{b}(i-j), \\
t_{s, a}(i-j) & =2 t_{s, p} \bar{\lambda}_{a}(i-j), \\
t_{s, b}(i-j) & =2 t_{s, p} \bar{\lambda}_{b}(i-j), \\
t_{a, a}(i-j) & =-t_{b, b}(i-j)=-4 t_{p, p} \tau(i-j), \\
t_{d, a} & =2 t_{d, p} \lambda_{a}(0), \\
\epsilon_{a, b} & =\epsilon_{p} \pm 4 t_{p, p} \tau(0)
\end{aligned}
$$


(функция $\tau(\vec{r})$ приведена в работах [6]).

Таким образом, мы закончили преобразование всех членов гамильтониана (1) к ячеечному (кластерному) представлению. В гамильтониане (4) выделена в качестве главной локальная часть (5), которая содержит лишь фермионы, локализованные на узлах меди.

Вторая часть гамильтониана, определенная выражением (6), состоит из вкладов межъячеечного туннелирования фермионов Ванье. Она содержит уменьшающиеся с расстоянием матричные элементы квантовых переходов, которые рассматриваются в дальнейшем как возмущение основного локального вклада.

Возмущение $H^{\prime}$ имеет довольно сложный вид, так как содержит несколько различных межъячеечных механизмов туннелирования.

В дальнейшем сначала будут исследованы (следуя работам [6], [7]) свойства локального гамильтониана. Затем будет рассмотрен процесс делокализации фермионов на основе термодинамической теории возмущений для сильно коррелированных систем, развитой в работах [8], [9].

\section{3. ЭЛЕКТРОННЫЙ СПЕКТР В АТОМНОМ ПРЕДЕЛЕ}

Рассматриваемая система с гамильтонианом нулевого приближения (5) состоит из двух подсистем. Одна подсистема из $d$ - и $a$-фермионов является сильно коррелированной из-за кулоновского отталкивания $d$-электронов и гибридизации $d$ - и $a$-орбиталей. Вторая подсистема состоит из свободных $b$ - и $s$-фермионов.

Диагонализация локального гамильтониана коррелированной $(d, a)$-подсистемы фермионов может быть осуществлена на основе специального метода канонического преобразования, разработанного для исследования периодической модели Андерсона и трехзонной модели Хаббарда в работах [6], [7].

Перечислим все шестнадцать исходных состояний коррелированной $(d, a)$-подсистемы:

$$
\begin{array}{lll}
\Psi_{1}=|0\rangle, & \Psi_{9}=a_{\uparrow}^{+} d_{\uparrow}^{+}|0\rangle, \\
\Psi_{2}=a_{\uparrow}^{+}|0\rangle, & \Psi_{10}=\frac{1}{\sqrt{2}}\left(a_{\uparrow}^{+} d_{\downarrow}^{+}+a_{\downarrow}^{+} d_{\uparrow}^{+}\right)|0\rangle, \\
\Psi_{3}=d_{\uparrow}^{+}|0\rangle, & \Psi_{11}=a_{\downarrow}^{+} d_{\downarrow}^{+}|0\rangle, \\
\Psi_{4}=a_{\downarrow}^{+}|0\rangle, & \Psi_{12}=d_{\uparrow}^{+} a_{\uparrow}^{+} a_{\downarrow}^{+}|0\rangle, \\
\Psi_{5}=d_{\downarrow}^{+}|0\rangle, & \Psi_{13}=a_{\uparrow}^{+} d_{\uparrow}^{+} d_{\downarrow}^{+}|0\rangle, \\
\Psi_{6}=a_{\uparrow}^{+} a_{\downarrow}^{+}|0\rangle, & \Psi_{14}=d_{\downarrow}^{+} a_{\uparrow}^{+} a_{\downarrow}^{+}|0\rangle, \\
\Psi_{7}=\frac{1}{\sqrt{2}}\left(a_{\uparrow}^{+} d_{\downarrow}^{+}-a_{\downarrow}^{+} d_{\uparrow}^{+}\right)|0\rangle, & \Psi_{15}=a_{\downarrow}^{+} d_{\uparrow}^{+} d_{\downarrow}^{+}|0\rangle, \\
\Psi_{8}=d_{\uparrow}^{+} d_{\downarrow}^{+}|0\rangle, & \Psi_{16}=a_{\uparrow}^{+} a_{\downarrow}^{+} d_{\uparrow}^{+} d_{\downarrow}^{+}|0\rangle,
\end{array}
$$

где $|0\rangle$ есть вакуумное состояние.

В результате кулоновского отталкивания и $d-a$-гибридизации происходит перенормировка состояний (7) и их энергий. Возникающие вновь состояния, обозначаемые через $\left|E_{\lambda}\right\rangle$, с собственным значением энергии $E_{\lambda}$, где $\lambda=1, \ldots, 16$, связаны с 
исходными состояниями (7) следующими соотношениями [6]:

$$
\Psi_{n}=\sum_{\lambda} \Phi_{n}\left(E_{\lambda}\right)\left|E_{\lambda}\right\rangle, \quad\left|E_{\lambda}\right\rangle=\sum_{n} \Phi_{n}^{*}\left(E_{\lambda}\right) \Psi_{n}
$$

где $\Phi_{n}\left(E_{\lambda}\right)$ - полная система ортонормированных функций, являющихся собственными функциями матрицы локального гамильтониана $(d, a)$-подсистемы.

Приведем простейшие примеры. В связи с тем, что вакуумное состояние не ренормируется, собственными состояниями являются состояния с числом частиц, равным единице, спином $S=1 / 2$ и двумя его проекциями $S_{z}= \pm 1 / 2$. Непереномированные состояния $\Psi_{2}$ и $\Psi_{3}$ в результате гибридизации преобразуются в связанное $\left(\left|E_{2}\right\rangle\right)$ и антисвязанное $\left(\left|E_{3}\right\rangle\right)$ дублетные состояния вида

$$
\left|E_{2}\right\rangle=\Phi_{2}\left(E_{2}\right) \Psi_{2}+\Phi_{3}\left(E_{2}\right) \Psi_{3}, \quad\left|E_{3}\right\rangle=\Phi_{2}\left(E_{3}\right) \Psi_{2}+\Phi_{3}\left(E_{3}\right) \Psi_{3}
$$

с ренормированными значениями энергии $\left(t_{d, a} \equiv V\right)$

$$
E_{2,3}=\frac{1}{2}\left[\epsilon_{a}+\epsilon_{d} \mp \sqrt{\left(\epsilon_{a}-\epsilon_{d}\right)^{2}+4 V^{2}}\right]
$$

и диагонализующими функциями

$$
\begin{aligned}
& \Phi_{2}\left(E_{2,3}\right)=\frac{1}{\sqrt{2}}\left[1 \pm \frac{\epsilon_{a}-\epsilon_{d}}{\sqrt{\left(\epsilon_{a}-\epsilon_{d}\right)^{2}+4 V^{2}}}\right]^{1 / 2}, \\
& \Phi_{3}\left(E_{2,3}\right)=\mp \operatorname{Sgn}(V) \frac{1}{\sqrt{2}}\left[1 \mp \frac{\epsilon_{a}-\epsilon_{d}}{\sqrt{\left(\epsilon_{a}-\epsilon_{d}\right)^{2}+4 V^{2}}}\right]^{1 / 2} .
\end{aligned}
$$

Состояния $\left|E_{4}\right\rangle$ и $\left|E_{5}\right\rangle$ имеют обратную проекцию спина, а в остальном совпадают с $\left|E_{2}\right\rangle$ и $\left|E_{3}\right\rangle$.

Рассмотрим теперь состояния с двумя частицами и двумя возможными значениями спина $S=0$ и $S=1$. При $S=0$ имеются три синглетных состояния $\left|E_{\lambda}\right\rangle$ со значениями $\lambda=6,7,8$. Функции этих состояний связаны с неперенормированными соотношениями

$$
\left|E_{\lambda}\right\rangle=\sum_{n=6,7,8} \Phi_{n}\left(E_{\lambda}\right) \Psi_{n}, \quad \Psi_{n}=\sum_{\lambda=6,7,8} \Phi_{n}\left(E_{\lambda}\right)\left|E_{\lambda}\right\rangle .
$$

Три значения энергии определяются из уравнения

$$
\left(2 \epsilon_{a}-E\right)\left(\epsilon_{a}+\epsilon_{d}-E\right)\left(2 \epsilon_{d}+U-E\right)-4 V^{2}\left(\epsilon_{a}+\epsilon_{d}+\frac{U}{2}-E\right)=0 .
$$

В пределе большого значения кулоновского отталкивания $U \gg|V|$ энергия синглета Жанга-Райса имеет вид

$$
E_{6} \simeq \frac{3 \epsilon_{a}+\epsilon_{d}}{2}-\sqrt{\left(\frac{\epsilon_{a}-\epsilon_{d}}{2}\right)^{2}+2 V^{2}}-\frac{V^{2}}{U}\left[1+\frac{\epsilon_{a}-\epsilon_{d}}{\sqrt{\left(\epsilon_{a}-\epsilon_{d}\right)^{2}+8 V^{2}}}\right]
$$


Энергия трех двухчастичных триплетных состояний не ренормируется и равна $E_{9}=$ $\epsilon_{a}+\epsilon_{d}$. Разность энергий $\Delta E=E_{9}-E_{6}$ определяет ту эффективную температуру, при которой система может перейти из первоначально синглетного в конечное триплетное состояние с изменением ее магнитных свойств.

Между шестнадцатью квантовыми исходными состояниями ячейки имеются десять квантовых переходов с изменением числа частиц на 1 и значения проекции спина на $1 / 2$.

На основании формул (7) определим операторы Хаббарда квантовых переходов $X^{m n}=\left|\Psi_{m}\right\rangle\left\langle\Psi_{n}\right|$ и установим связь между ними и фермионными операторами коррелированной подсистемы:

$$
\begin{aligned}
d_{\uparrow}=X^{1,3}-X^{2,9}+X^{5,8}+\frac{1}{\sqrt{2}}\left(X^{4,7}-X^{4,10}\right)+ \\
+X^{6,12}-\frac{1}{\sqrt{2}}\left(X^{7,13}+X^{10,13}\right)-X^{11,15}+X^{14,16}, \\
a_{\uparrow}=X^{1,2}+X^{4,6}+X^{3,9}+\frac{1}{\sqrt{2}}\left(X^{5,7}+X^{5,10}\right)+ \\
\quad+X^{8,13}+\frac{1}{\sqrt{2}}\left(X^{10,12}-X^{7,12}\right)+q X^{11,14}+X^{15,16} .
\end{aligned}
$$

Обозначим через $Y^{\lambda, \lambda^{\prime}}=\left|E_{\lambda}\right\rangle\left\langle E_{\lambda^{\prime}}\right|$ оператор Хаббарда для квантовых переходов между ренормированными состояниями ячеечного представления. На основании соотношений (8) легко установить связь между операторами $X^{m n}$ и $Y^{\lambda \lambda^{\prime}}$ :

$$
\begin{aligned}
X^{m n} & =\sum_{\lambda, \lambda^{\prime}} \Phi_{m}\left(E_{\lambda}\right) \Phi_{n}\left(E_{\lambda^{\prime}}\right) Y^{\lambda \lambda^{\prime}}, \\
Y^{\lambda \lambda^{\prime}} & =\sum_{m, n} \Phi_{m}\left(E_{\lambda}\right) \Phi_{n}\left(\lambda^{\prime}\right) X^{m n} .
\end{aligned}
$$

Исходя из формул (9) и (10) легко вычислить двухвременные одно- и двухчастичные пропагаторы ячейки. Приведем в качестве примера пропагатор одного из синглетов:

$$
G_{\sigma}^{(0)}\left(\tau-\tau^{\prime}\right)=\frac{1}{2}\left\langle T\left(a_{-\sigma} d_{\sigma}-a_{\sigma} d_{-\sigma}\right)_{\tau}\left(\bar{d}_{\sigma} \bar{a}_{-\sigma}-\bar{d}_{-\sigma} \bar{a}_{\sigma}\right)_{\tau^{\prime}}\right\rangle_{0} .
$$

Его фурье-образ имеет вид $(\Omega=2 m \pi / \beta)$

$$
\begin{aligned}
G_{\sigma}^{0}(i \Omega)= & \frac{1}{Z_{0}} \sum_{a=6}^{8} \Phi_{7}\left(E_{a}\right)^{2}\left[\frac{e^{-\beta E_{a}}-e^{-\beta E_{1}}}{i \Omega+E_{1}-E_{a}}+\frac{e^{-\beta E_{16}}-e^{-\beta E_{a}}}{i \Omega+E_{a}-E_{16}}\right]+ \\
& +\frac{1}{Z_{0}} \sum_{\lambda=2,3} \sum_{\lambda^{\prime}=12,13}\left[\Phi_{2}\left(E_{\lambda}\right) \Phi_{12}\left(E_{\lambda^{\prime}}\right)+\Phi_{3}\left(E_{\lambda}\right) \Phi_{13}\left(E_{\lambda^{\prime}}\right)\right]^{2} \frac{e^{-\beta E_{\lambda^{\prime}}}-e^{-\beta E_{\lambda}}}{i \Omega+E_{\lambda}-E_{\lambda^{\prime}}},
\end{aligned}
$$

где $Z_{0}=\sum_{\lambda=1}^{16} e^{-\beta E_{\lambda}}$. Энергетические знаменатели в формуле (11) содержат разности энергий состояний с одинаковыми значениями спина и его проекции, но с отличающимся на два числом частиц. На основании формул (9) и (10) можно определить и другие пропагаторы ячейки. 


\section{4. ТЕОРИЯ ВОЗМУЩЕНИЙ}

Процесс делокализации кластерных фермионов определяется их туннелированием между узлами меди. Этот процесс не ограничивается ближайшими соседями, как это предполагалось в исходном гамильтониане (1). Переход к ячеечному представлению привел к дальнодействию перескоков, как это видно из структуры функций $\lambda_{a}, \lambda_{b}, \bar{\lambda}_{a}$ и $\bar{\lambda}_{b}$, которые, однако, уменьшаются с расстоянием.

Как отмечалось выше, благодаря присутствию сильного кулоновского отталкивания $d$-электронов и их гибридизации с $a$-электронами подсистема $(d, a)$-электронов сильно коррелирована, и для нее следует применить теорию возмущений, развитую в работах [8] для нормального и в работах [9] для сверхпроводящего состояний. Для подсистемы $(s, b)$-фермионов справедлива обычная теория возмущений статистической механики.

Рассмотрим перенормированные функции Грина делокализованных фермионов $(x=\vec{x}, \sigma, \tau)$ :

$$
\begin{aligned}
G_{d, d}\left(x \mid x^{\prime}\right) & =-\left\langle T d(x) \bar{d}\left(x^{\prime}\right) U(\beta)\right\rangle_{0}^{\mathrm{c}}, \\
G_{a, d}\left(x, x^{\prime}\right) & =-\left\langle T a(x) \bar{d}\left(x^{\prime}\right) U(\beta)\right\rangle_{0}^{\mathrm{c}}, \\
G_{s, s}\left(x, x^{\prime}\right) & =-\left\langle T s(x) \bar{s}\left(x^{\prime}\right) U(\beta)\right\rangle_{0}^{\mathrm{c}},
\end{aligned}
$$

где $U(\beta)$ - оператор эволюции, определенный возмущением $H^{\prime}$. Индекс "с" указывает на связность диаграмм. Аналогичным образом определяются и другие пропагаторы системы. В сверхпроводящем состоянии системы наряду с функциями (12) следует рассмотреть квазисредние Боголюбова в виде аномальных функций Грина:

$$
\begin{aligned}
F_{d, d}\left(x, x^{\prime}\right) & =-\left\langle T d(x) d\left(x^{\prime}\right) U(\beta)\right\rangle_{0}^{c}, \\
\bar{F}_{d, d}\left(x, x^{\prime}\right) & =-\langle T \bar{d}(x) \bar{d} U(\beta)\rangle_{0}^{\mathrm{c}}, \\
F_{a, d}\left(x, x^{\prime}\right) & =-\left\langle\operatorname{Ta}(x) d\left(x^{\prime}\right) U(\beta)\right\rangle_{0}^{c} .
\end{aligned}
$$

Уравнения Дайсона для этих и других (не выписанных) функций Грина, могут быть получены аналогично тому, как это было выполнено в работах [8], [9] для однозонной модели Хаббарда. Особенность рассматриваемой нами четырехзонной модели состоит в существовании не одного, а нескольких механизмов туннелирования, в которых участвуют все четыре орбитали. Поэтому новые уравнения должны учитывать это многообразие. Так же, как и в однозонном случае, удобно воспользоваться фурье-представлением как по пространственным, так и по временнь́м переменным пропагаторов. Для краткости записи используется четырехкомпонентнный вектор $(k=\vec{k}, i \omega)$, где $\omega$ - нечетная мацубаровская частота. Все функции Грина отмечены индексами тех фермионов, движение которых они описывают. Этими индексами отмечены также корреляционные функции

$$
\begin{aligned}
& \Lambda_{d, d}(k)=G_{d, d}^{0}(k)+Z_{d, d}(k), \\
& \Lambda_{a, d}(k)=G_{a, d}^{0}(k)+Z_{a, d}(k)
\end{aligned}
$$

и параметры порядка $Y_{d, d}(k), Y_{a, d}(k), \bar{Y}_{d, d}(k)$ и $\bar{Y}_{a, d}(k)$ сверхпроводящей фазы системы. Отметим также, что нами рассматривается синглетная сверхпроводимость, и 
поэтому спиновая зависимость аномальных величин имеет вид $F_{\sigma,-\sigma}, Y_{\sigma,-\sigma}, \bar{F}_{-\sigma, \sigma}$ и $\bar{Y}_{-\sigma, \sigma}$. Сверхпроводимость реализуется на фоне парамагнитной фазы системы, поэтому спиновую зависимость нормальных функций Грина можно опустить.

После этих предварительных замечаний приведем систему уравнений типа Дайсона для делокализованных пропагаторов коррелированной системы. Первое из них имеет вид

$$
\begin{aligned}
& G_{d, d}(k)=\Lambda_{d, d}(k)+\Lambda_{d, d}(k) t_{d, a}(\vec{k}) G_{a, d}(k)+ \\
&+\Lambda_{d, d}(k) t_{d, b}(\vec{k}) G_{b, d}(k)+\Lambda_{d, a}(k) t_{a, d}(\vec{k}) G_{d, d}(k)+ \\
&+\Lambda_{d, a}(k) t_{a, a}(\vec{k}) G_{a, d}(k)+\Lambda_{d, a}(k) t_{a, s}(\vec{k}) G_{s, d}(k)- \\
&-Y_{d, d}(k) t_{a, d}(-\vec{k}) \bar{F}_{a, d}(k)-Y_{d, d}(k) t_{b, d}(-\vec{k}) \bar{F}_{b, d}(k)- \\
&-Y_{d, a}(k) t_{d, a}(-\vec{k}) \bar{F}_{d, d}(k)-Y_{d, a}(k) t_{a, a}(-\vec{k}) \bar{F}_{a, d}(k)- \\
&-Y_{d, a}(k) t_{s, a}(-\vec{k}) \bar{F}_{s, d}(k) .
\end{aligned}
$$

Замена в уравнении (15) левого фермионного индекса $d$ на $a$ приведет к уравнению для функции $G_{a, d}(k)$. Уравнение для одной из аномальных функций Грина имеет вид

$$
\begin{aligned}
& \bar{F}_{d, d}(k)=\bar{Y}_{d, d}(k)+\bar{Y}_{d, d}(k) t_{d, a}(\vec{k}) G_{a, d}(k)+ \\
&+\bar{Y}_{d, d}(k) t_{d, b}(\vec{k}) G_{b, d}(k)+\bar{Y}_{d, a}(k) t_{a, d}(\vec{k}) G_{d, d}(k)+ \\
&+\bar{Y}_{d, a}(k) t_{a, a}(\vec{k}) G_{a, d}(k)+\bar{Y}_{d, a}(k) t_{a, s}(\vec{k}) G_{s, d}(k)+ \\
&+\Lambda_{d, d}(-k) t_{a, d}(-\vec{k}) \bar{F}_{a, d}(k)+\Lambda_{d, d}(-k) t_{b, d}(-\vec{k}) \bar{F}_{b, d}(k)+ \\
&+\Lambda_{d, a}(-k) t_{d, a}(-\vec{k}) \bar{F}_{d, d}(k)+\Lambda_{d, a}(-k) t_{a, a}(-\vec{k}) \bar{F}_{a, d}(k)+ \\
&+\Lambda_{d, a}(-k) t_{s, a}(-\vec{k}) \bar{F}_{s, d}(k) .
\end{aligned}
$$

Уравнение для функции $\bar{F}_{a, d}(k)$ также получается из вышеприведенного уравнения (16) заменой левого фермионого индекса.

Благодаря туннелированию между орбиталями сильно коррелированных и не коррелированных фермионов в вышеприведенных уравнениях появились смешанные пропагаторы вида $G_{s, d}(k), G_{b, d}(k), \bar{F}_{s, d}(k), \bar{F}_{b, d}(k)$. Для этих функций Грина также можно написать уравнения типа Дайсона:

$$
\begin{aligned}
G_{b, d}(k)= & \frac{G_{b, b}(k)^{0}}{d(k)}\left[t_{b, d}(\vec{k}) G_{d, d}(k)+t_{s, b}(\vec{k}) G_{s, s}(k)^{0} G_{a, d}(k)\right] \\
G_{s, d}(k)= & \frac{G_{s, s}(k)^{0}}{d(k)}\left[\left(1-t_{b, b}(\vec{k}) G_{b, b}(k)^{0}\right) G_{a, d}(k)+t_{s, b}(\vec{k}) G_{b, b}(k)^{0} t_{b, d}(\vec{k}) G_{d, d}(k)\right], \\
\bar{F}_{b, d}(k)= & \frac{G_{b, b}(-k)^{0}}{d(-k)}\left[t_{d, b}(-\vec{k}) \bar{F}_{d, d}(k)+t_{s, b}(-\vec{k}) G_{s, s}(-k)^{0} t_{a, s}(-\vec{k}) \bar{F}_{a, d}(k)\right] \\
\bar{F}_{s, d}(k)= & \frac{G_{s, s}(-k)^{0}}{d(-k)}\left[\left(1-t_{b, b}(\vec{k}) G_{b, b}(k)^{0}\right) t_{a, s}(-\vec{k}) \bar{F}_{a, d}(k)+\right. \\
& \left.\quad+t_{b, s}(-\vec{k}) G_{b, b}(-k)^{0} t_{d, b}(-\vec{k}) \bar{F}_{d, d}(k)\right]
\end{aligned}
$$


где

$$
d(k)=1-G_{b, b}(k)^{0} t_{b, b}(\vec{k})-G_{s, s}(k)^{0} t_{s, b}(k) G_{b, b}(k)^{0} t_{b, s}(\vec{k}) .
$$

Если подставить выражения для смешанных функций Грина (17) в уравнения (15) и (16), то получим более простую систему уравнений для перенормированных функций Грина $G_{d, d}$ и $G_{a, d}$, а также $\bar{F}_{d, d}$ и $\bar{F}_{a, d}$, сильно коррелированной подсистемы. Эти уравнения содержат перенормированные матричные элементы туннелирования, которые определяются равенствами

$$
\begin{aligned}
& \bar{t}_{a, a}(k)=t_{a, a}(\vec{k})+\frac{1}{d(k)} t_{a, s}(\vec{k}) G_{s, s}(k)^{0}\left(1-t_{b, b}(\vec{k}) G_{b, b}(k)^{0}\right) t_{s, a}(\vec{k}), \\
& \bar{t}_{a, d}(k)=t_{a, d}(\vec{k})+\frac{1}{d(k)} t_{a, s}(\vec{k}) G_{s, s}(k)^{0} t_{s, b}(\vec{k}) G_{b, b}(k)^{0} t_{b, d}(\vec{k}) \\
& \bar{t}_{d, a}(k)=t_{d, a}(\vec{k})+\frac{1}{d(k)} t_{d, b}(\vec{k}) G_{b, b}(k)^{0} t_{b, s}(\vec{k}) G_{s, s}(k)^{0} t_{s, a}(\vec{k}) \\
& \bar{t}_{d, d}(k)=\frac{1}{d(k)} t_{d, b}(\vec{k}) G_{b, b}(k)^{0} t_{b d}(\vec{k})
\end{aligned}
$$

Введем новые обозначения

$$
\begin{aligned}
L_{\alpha, \beta}(k) & =\Lambda_{\alpha, a}(k) \bar{t}_{a, \beta}(k)+\Lambda_{\alpha, d}(k) \bar{t}_{d, \beta}(k), \\
L_{\alpha, \beta}(-k) & =\Lambda_{\alpha, a}(k) \bar{t}_{\beta, a}(-k)+\Lambda_{\alpha, d}(k) \bar{t}_{\beta, d}(-k), \\
K_{\alpha, \beta}(k) & =Y_{\alpha, a}(k) \bar{t}_{\beta, a}(-k)+Y_{\alpha, d}(k) \bar{t}_{\beta, d}(-k), \\
\bar{K}_{\alpha, \beta}(k) & =\bar{Y}_{\alpha, a}(k) \bar{t}_{a, \beta}(k)+\bar{Y}_{\alpha, d}(k) \bar{t}_{d, \beta}(k) .
\end{aligned}
$$

В этих обозначениях уравнения Дайсона для перенормированных функций Грина сильно коррелированной подсистемы имеют простой вид:

$$
\begin{aligned}
& G_{d, d}(k)\left(1-L_{d, d}(k)\right)-L_{d, a}(k) G_{a, d}(k)+ \\
& \quad+K_{d, a}(k) \bar{F}_{a, d}(k)+K_{d, d}(k) \bar{F}_{d, d}(k)=\Lambda_{d, d}(k), \\
& G_{a, d}(k)\left(1-L_{a, a}(k)\right)-L_{a, d}(k) G_{d, d}(k)+ \\
& \quad+K_{a, a}(k) \bar{F}_{a, d}(k)+K_{a, d}(k) \bar{F}_{d, d}(k)=\Lambda_{a, d}(k), \\
& \bar{F}_{d, d}(k)\left(1-L_{d, d}(-k)\right)-L_{d, a}(-k) \bar{F}_{a, d}(k)- \\
& \quad-\bar{K}_{d, a}(k) G_{a, d}(k)-\bar{K}_{d, d}(k) G_{d, d}(k)=\bar{Y}_{d, d}(k), \\
& \bar{F}_{a, d}(k)\left(1-L_{a, a}(-k)\right)-L_{a, d}(-k) \bar{F}_{d, d}(k)- \\
& \quad-\bar{K}_{a, d}(k) G_{d, d}(k)-\bar{K}_{a, a}(k) G_{a, d}(k)=\bar{Y}_{a, d}(k) .
\end{aligned}
$$

Аналогичным образом определяются остальные функции Грина коррелированной подсистемы $\left(G_{a, a}, G_{d, a}, \bar{F}_{a, a}, \bar{F}_{d, a}\right)$. Согласно этим уравнениям, перенормированные функции Грина выражаются через перенормированные корреляционные функции $Z, Y, \bar{Y}$, которые представляют собой бесконечные последовательности диаграмм, содержащих неприводимые корреляционные функции Грина. Для корреляционных функций не существует уравнений типа Дайсона. Поэтому для получения полной системы уравнений, позволяющей определить все искомые величины, следует ограничиться определенным классом диаграмм и замкнуть тем самым систему уравнений. 
Несмотря на то что $s$ - и $b$-фермионы не коррелированы, их совместное участие в процессах туннелирования с коррелированными $d$ - и $a$-фермионами приводит к тому, что их пропагаторы также определяются сильными корреляциями. Обратное влияние фермионов было продемонстрировано формулами для перенормирванных матричных элементов межузельного туннелирования.

В нормальном состоянии системы аномальные функции Грина равны нулю, и перенормированные пропагаторы сильно коррелированной подсистемы имеют вид

$$
\begin{aligned}
G_{d, d}(k) & =\frac{1}{D(k)}\left[\Lambda_{d, d}(k)+\left(\Lambda_{a, d}(k) L_{d, a}(k)-\Lambda_{d, d}(k) L_{a, a}(k)\right)\right], \\
G_{a, d}(k) & =\frac{1}{D(k)}\left[\Lambda_{a, d}(k)-\left(\Lambda_{a, d}(k) L_{d, d}(k)-\Lambda_{d, d}(k) L_{a, d}(k)\right)\right], \\
D(k) & =\left(1-L_{a, a}(k)\right)\left(1-L_{d, d}(k)\right)-L_{a, d}(k) L_{d, a}(k) .
\end{aligned}
$$

Если предположить симметрию двухкомпонентной коррелированной подсистемы в смысле выполнения равенств $G_{\alpha, \beta}=G, F_{\alpha, \beta}=F, Y_{\alpha, \beta}=Y$ и т.д., где $(\alpha, \beta)=$ $(d, a)$, то можно получить простые формулы

$$
\begin{aligned}
G(k) & =\frac{1}{D(k)}[\Lambda(k)(1-\Lambda(-k) \bar{t}(-k))-\bar{t}(-k) Y(k) \bar{Y}(k)], \\
F(k) & =\frac{Y(k)}{D(k)}, \quad \bar{F}(k)=\frac{\bar{Y}(k)}{D(k)}, \\
D(k) & =(1-\bar{t}(k) \Lambda(k))(1-\bar{t}(-k) \Lambda(-k))+\bar{t}(k) \bar{t}(-k) Y(k) \bar{Y}(k),
\end{aligned}
$$

где

$$
\bar{t}(k)=\bar{t}_{a, a}(k)+\bar{t}_{a, d}(k)+\bar{t}_{d, a}(k)+\bar{t}_{d, d}(k) .
$$

Эти равенства соответствуют динамике однозонной модели Хаббарда с эффективным динамическим матричным элементом туннелирования $\bar{t}(k)$. Следует заметить, что экспериментальное исследование многозонных купратов обнаруживает однозонные черты, и приведенное выше симметричное решение может помочь в понимании этого свойства. Дальнейшее исследование симметричного варианта теории должно опираться на результаты работ, посвященных однозонной модели Хаббарда.

\section{5. ЗАКЛЮЧЕНИЕ}

Четырехзонная модель Хаббарда, построенная на двух орбиталях $\left(3 d_{x^{2}-y^{2}}, 4 s\right)$ меди и двух орбиталях $\left(2 p_{x}, 2 p_{y}\right)$ кислорода, преобразуется в ячеечную модель, ортогонализованную на решетке меди. В основе преобразования лежит введение коллективных кислородных мод. Новый гамильтониан состоит из основной локальной части и возмущения, возникающего из-за процессов туннелирования всех ортогонализованных фермионных мод между узлами решетки меди.

В работе частично выполнена диагонализация локального гамильтониана и определены низко лежащие (по энергиям) состояния, а именно одночастичные дублетные и двухчастичные синглетные и триплетные состояния. Синглет Жанга-Райса имеет минимальное значение энергии среди двухчастичных состояний. 
Развита термодинамическая теория возмущений сильно коррелированной системы. В качестве нулевого приближения этой теории выступает локальная кластерная модель четырехзонных купратов, а в качестве возмущения - межкластерное туннелирование фермионов. Выполнены необходимые обобщения диаграммной техники, вытекающие из существования сильного кулоновского отталкивания. Установлены уравнения типа Дайсона для основных пропагаторов данной модели и получен простой симметричный вариант данной теории.

Благодарности. Авторы (В. А. Москаленко, Н. М. Плакида и П. Ентел) выражают признательность за предоставление грантов Программы Гейзенберг-Ландау. Один из авторов (В. А. Москаленко) благодарит университет Дуйсбург-Эссен за гостеприимство и финансовую поддержку. П. Ентел благодарит лабораторию теоретической физики им. Н.Н. Боголюбова ОИЯИ за гостеприимство во время его пребывания в Дубне.

\section{Список литературы}

[1] Н. М. Плакида, Высокотемпературные сверхпроводники, Международная программа образования, M., 1996; D. J. Scalapino, Phys. Rep., 250 (1995), 329; V. Yu. Yushankhai, V.S. Oudovenko, R. Hayn, Phys. Rev. B, 55 (1997), 15562; N. M. Plakida, R. Hayn, J.-L. Richard, Phys. Rev. B, 51 (1995), 16599; Н. М. Плакида, Л. Антон, С. Адам, Г. Адам, ЖЭТФ, 124 (2003), 367; cond-mat/0104234.

[2] T. M. Mishonov, J. P. Wallington, E. S. Penev, J. O. Indekeu, Mod. Phys. Lett. B, 16 (2002), 693; T. M. Mishonov, A. A. Donkov, R. K. Koleva, E.S. Penev, Bulgarian J. Phys., 24:3/4 (1997), 114; cond-mat/0001033.

[3] T. M. Mishonov, J. O. Indekeu, E. S. Penev, Int. J. Mod. Phys. B, 16 (2002), 4577; J. Phys.: Cond. Mat., 15 (2003), 4429.

[4] F. C. Zhang, T. M. Rice, Phys. Rev. B, 37 (1988), 3759.

[5] V. J. Emery, G. Reiter, Phys. Rev. B, 38 (1988), 4547.

[6] В. А. Москаленко, П. Ентел, М. Маринаро, Д. Ф. Дигор, Д. Греку, ЭЧАЯ, 33 (2002), 964; В. А. Москаленко, Н. Б. Перкинс, ТМФ, 121 (1999), 464.

[7] V. A. Moskalenko, P. Entel, M. Marinaro, N. B. Perkins, C. Holtfort, Phys. Rev. B, 63 (2001), 245119.

[8] М. И. Владимир, В.А. Москаленко, ТМФ, 83 (1990), 428; С.И. Вакару, М.И. Владимир, В.А. Москаленко, ТМФ, 85 (1990), 248.

[9] Н. Н. Боголюбов, В. А. Москаленко, ТМФ, 86:1 (1991), 16; 92 (1992), 182; Краткие Сообщения ОИЯИ, 5/44 (1990), 3-28; ДАН СССР, 316 (1991), 1107. 